\title{
FRANS-CREOOLSE VERSJES VAN GUADELOUPE
}

\author{
GEPUBLICEERD DOOR
}

\author{
W. DE Barbanson O.P. (St. Maarten)
}

Er zijn op Guadeloupe en Martinique genoeg Creoolse gezangen te krijgen, met muziek en al uitgegeven: producten van plaatselijke dichters en componisten, die hun uitgaven beschermen met een verbod van nadruk. Werkelijke volksliedjes zijn déze echter meestal niet geworden.

De meeste van de hier volgende liedjes van Guadeloupe spreken voor zich zelf. Een enkele opmerking moge het begrijpen ervan den lezer wat gemakkelijker maken.

In het algemeen accentueren de Creolen de neusklanken nog sterker dan de Fransen. Dat is misschien de verklaring van het feit dat de Creolen een soort vereenvoudigde spelling gebruiken, zoals couven voor couvent. Ook de bewoners van Frankrijk spreken de laatste letter niet uit, maar een zekere slordigheid van spreken en schrijven is er hier toch wel waar te nemen. Vooral medeklinkers worden daarvan nog wel eens het slachtoffer, zoals coù̀ - croire, pède - perdre, cò - corps. Het zijn echter niet alleen de medeklinkers die mishandeld worden, óók de klinkers; men lette b.v. op het einde van het eerste versje: $d u$ quatrevingt-dix ans, in plaats van de quatre-vingt-dix ans.

Voor de geslachten der woorden hebben de Creolen weinig gevoel: $u n$ en une is hun vrijwel hetzelfde; het is even goed en jeune fiyète (une jeune fille) als en ange (un ange). Ook gebruiken ze yon voor un en une.

Als bezittelijk voornaamwoord gebruiken ze $l i$, dat achter het zelfstandig naamwoord wordt geplaatst: tête $l i$, paren $l i$, famille $l i$. Niet alleen wordt niet op de geslachten gelet, er is ook geen verschil tussen enkelvoud en meervoud. Mijn is moin, dit wordt ook achter het zelfstandig naamwoord gezet: jeune an moin (mes jeunes ans). Men denkt hier aan de Franse constructie, wanneer iets met nadruk gezegd wordt: ce livre est à moi, tegenover mon livre.

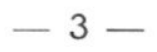


Moin kan in het creools ook het persoonlijk voornaamwoord $i k$ zijn. $\mathrm{Ca}$ (moet worden uitgesproken als $k a$, anders zou het ça moeten zijn) is hetzelfde als kaille; het dient om de toekomende wijs uit te drukken, b.v. ca mor (elle mourra), zij zal sterven.

En nu het eerste liedje.

\section{VOCATION TARDIVE.}

En jeune fiyète de quatre vingt

[dix ans

Une jeune fillette de quatre vingt

Metté en jou dan tête li D'entré dans en couven. En quittant famille li

Paren li ami li, Se mit un jour en tête

[dix ans

Li di a Marie guidé jeune an moin.

D'entrer dans un couvent.

En quittant sa famille

Ses parents, ses amis,

2

$\mathrm{Y}$ entré $\mathrm{o}$ monastère, monastère

[cloitré,

Y ca di a la Mère:

Esce moin peu rentré?

Moin pa couè moin tro jeune?

Esce moin ni l'age voulu?

Esce yo ca permète jeune

Car jeune ca flétri?

Elle dit à Marie guidez mes

[jeunes ans.

Entrant au monastère, monastère

Elle dit à la Mère:

[cloitré,

Pourrai-je y entrer ?

Ne crois-je pas trop jeune?

Ai-je l'âge requis ?

Permettra-t-on le jeûne,

Car le jeune flétrit?

3

Enfin mi l'époque de la prise

[d'habit

Plaizi la sufoqué. [d'habit

$\mathrm{Y}$ couè pède l'espri li.

Y o abiyé li, yo aprété li.

Cette jeune soeu la

Yo mété a sou tête li

En couronne de fleu.

Enfin voici l'époque de la prise

Le plaisir la suffoque.

[d'habit.

Elle croit perdre son esprit.

On l'habille, on l'apprète.

Cette jeune soeur

On lui met sur la tête

Une couronne de fleurs.

4

Y atrapé en rhumatisme,

Qui tienne toute co a li.

Y atrapé en anévrisme,

qui pêcé coeu a li.

Toute cé religieuse la

Di, can li té ca mor

Nous ca pède en ange

Du quatre vingt dix ans.

Elle eut un rhumatisme,

Qui lui tint tout le corps.

Elle eut un anéurisme,

Qui lui perça le coeur.

Toutes les religieuses

Disaient, quand elle mourra:

Nous perdrons un jeune ange

De quatre vingt dix ans.

Het volgende versje gaat over een mousse, de naam voor de jongste scheepsjongen, die door iedereen gecommandeerd wordt om alle mogelijke werkjes op te knappen. Hij is de jongeling, die op onze 
schepen met de fraaie naam van ketelbink wordt vereerd. - Het $t i$ van het eerste couplet is een samengetrokken uitspraak van pitit (petit). Dit $t i$ is niet te verwarren met $t i$ het werkwoord zijn in zijn verschillende vervoegingen, zowel voor hij is als wij zijn of $i k$ ben. Hier komt ook naar voren: an papa, an manman voor un père, une mère. In het derde couplet vinden we mové, wat mauvaise betekent. Het zou dus eigenlijk moeten zijn: quand la mer est mauvaise; geen gebruikelijke combinatie in het Frans: - In het vierde couplet hebben we té voor était. - In het tweede refrein betekent brin weinig, een beetje. Vandaar het $t i$ brin (petit brin) voor klein beetje. - Opmerkelijk is het woord royal, in het eerste couplet; dit schijnt een reminiscentie uit het oude koninklijke koloniale tijdperk te zijn.

YoN PITI MOUSSE.

Moin cé en piti ti mousse

A bor en vésso royal.

E ti ven la poussé moin

Nor ou sud moin bien négal.

\section{(Refrain)}

Car an papa et an manman

Moin jamais connate l'amou.

Non pesonne a sou la tè,

Pesonne pa ca atanne retou moin.

$$
2
$$

Quan moin monté a bordage,

Ou i ti ni toute vié solda,

Yo toute ca tremblé pou lage moin;

Moin sèl, moin pa ca tremblé.

\section{3}

Quan lan mè la ti mové,

Cé matlo la ca fè voeu

A jounou, con moin ca prié

$\mathrm{Pa}$ pou moin, mé pou yo.

4

Quecque foi brillante et belle Zétoile ca brié au ciel,

Yon voi chérie ca aplé moin,

Yon larme té en zieu moin.

(2e refrain)

Oui en ho moin ca espéré

Rutrouve en ti brin d'amou,

Oui en ho la moin ni en manman;

Li seul ca atanne retou moin.

\section{UN PETIT MOUSSE.}

Je ne suis qu'un petit mousse

A bord d'un vaisseau royal.

Partout où le petit vent me

Nord ou sud, ça m'est égal. [pousse

\section{(Retrain)}

Car d'un père et d'une mère. Je n'ai point connu l'amour.

Non personne sur la terre,

Personne n'attend mon retour.

Quand je monte à l'abordage,

Où sont tous nos vieux soldats,

Chacun tremble pour mon âge;

Moi seul je ne tremble pas.

Quand la mer est en furie,

Les matelots font des voeux

A genoux, quand je prie

Pas pour moi, mais pour eux.

Quelque fois brillante et belle

Une étoile brille aux cieux,

Une voix chérie m'appelle,

Une larme était dans mes yeux.

(2e refrain)

Car en haut oui je l'espère

Retrouver un petit peu d'amour,

Car là-haut j'ai une mère;

Elle seule attend mon retour. 
In het nu volgende herdersliedje komt nog beter uit, dat te, ti of tini door elkaar gebruikte vormen zijn van het werkwoord $z i j n$, zowel in de tegenwoordige als in de verleden tijd. Hier komen wij ook on tegen, voor un of une. - Omzettingen van letters zijn natuurlijk niet zeldzaam; in plaats van fromage spreekt men van fonmage. Ditzelfde verschijnsel hoort men ook op Curaçao, waar werd gesproken van Hilter of zelfs van Hiltoe, om Hitler aan te duiden. - Verder ziet men nog dat elle en $i l$ in het creools $y$ is.

TE TINI ON BERGÈRE.

\section{1}

Té tini on bergère,

Ron, ron, ron piti patapon;

Té tini on bergère

Qui ca gadé mouton. (bis)

$$
2
$$

$\mathbf{Y}$ fai yon fonmage,

Ron, ron, ron piti patapon;

$\mathrm{Y}$ fai yon fonmage

Epi lète a mouton li. (bis)

\section{3}

A chate la qui ca gadé li,

Ron, ron, ron piti patapon;

A chate la qui ca gadé li

Epi on piti lè fripon. (bis)

\section{4}

Si ou mété patte ou,

Ron, ron ron piti patapon;

Si ou mété patte ou,

ou kaille trapé baton. (bis)

\section{5}

Y pas mété patte li,

Ron, ron, ron patapon;

Y pas mété patte li,

Min y mété menton y. (bis)

6

La bergère la en colè,

Ron, ron, ron piti patapon;

La bergère la en colè

Tué piti chaton li. (bis)

\section{7}

$\mathrm{Y}$ alé la confèce,

Ron, ron, ron piti patapon;

$\mathrm{Y}$ allé la confèce,
IL ÉTAIT UNE BERGÈRE.

Il était une bergère,

Ron, ron, ron petit patapon:

Il était une bergère

Qui gardait ses moutons. (bis)

Elle en fit un fromage,

Ron, ron, ron petit patapon;

Elle en fit un fromage

Du lait de ses moutons. (bis)

Au chat qui la regarde,

Ron, ron, ron petit patapon;

Au chat qui la regarde

D'un petit air fripon. (bis)

Si tu y mets la patte,

Ron, ron, ron petit patapon;

Si tu y mets la patte,

Tu auras du bâton. (bis)

Il n'y mit pas la patte,

Ron, ron, ron patapon;

Il n'y mit pas la patte,

Mais il y mit le menton. (bis)

La bergère en colère,

Ron, ron, ron petit patapon:

La bergère en colère

Tua son petit chaton. (bis)

Elle alla à confesse,

Ron, ron, ron petit patapon;

Elle alla à confesse, 
Mon Père, je m'accuse

D'avoué tué chaton moin. (bis)

8

Ma fille pou pénitence,

Ron, ron, ron piti patapon;

Ma fille pou pénitence

Ou kaille embrassé li. (bis)

Pénitence la té douce,

Ron, ron, ron patapon,

Pénitence la té douce,

Moin kaille recommencé. (bis)
Mon Père, je m'accuse

D'avoir tué mon chaton. (bis)

Ma fille pour pénitence,

Ron, ron, ron petit patapon:

Ma fille pour pénitence

Vous allez l'embrasser. (bis)

La pénitence est douce,

Ron, ron, ron patapon;

La pénitence est douce,

Je recommencerai. (bis)

Nu krijgen wij weer een prachtig voorbeeld van verwaarlozing van het geslacht van woorden; en belle chato voor un beau chateau. Het creoolse bail, of $b a$, betekent geven.

MoIN Ni EN BELle chato.

1

Moin ni en belle chato, Ma tan ti reli relo.

Moin ni en belle chato,

Ma tan ti reli relo

2

Ta nou là plu belle, Ma tan ti reli relo.

Ta nou là plu belle, Ma tan ti reli relo.

3

Et nous kail détrui li, Ma tan ti reli relo.

Et nous kail détrui li, Ma tan ti reli relo.

\section{4}

Qui manniè ou kail fait ? Ma tan ti reli relo.

Qui manniè ou kail fait? Ma tan ti reli relo.

5

Nous kail pren roche pa roche, Ma tan ti reli relo.

Nous kail pren roche pa roche, Ma tan ti reli relo.
J'Ai UN BEAU ChÂteau.

J'ai un beau château, Ma tan ti reli relo.

J'ai un beau château, Ma tan ti reli relo.

Le nôtre est plus beau, Ma tan ti reli relo.

Le nôtre est plus beau, Ma tan ti reli relo.

Et nous le détruirons, Ma tan ti reli relo.

Et nous le détruirons, Ma tan ti reli relo.

Quelle manière ferez-vous? Ma tan ti reli relo.

Quelle manière ferez-vous? Ma tan ti reli relo.

Nous prendrons pierre par pierre, Ma tan ti reli relo.

Nous prendrons pierre par pierre, Ma tan ti reli relo. 
6

Et qui lèse ou kail pren?

Ma tan ti reli relo.

Et qui lèse ou kail pren?

Ma tan ti reli relo.

7

Nous kail pren manzelle $\mathrm{X}$,

$\mathrm{Ma}$ ta ti reli relo.

Nous kail pren manzelle X,

Ma tan ti reli relo.

8

Et qui métié ou kail bail ?

Mi ta ti reli relo.

Et qui métié ou kail bail ?

Mi tan ti reli relo.

\section{9}

Métié du repasseuse.

Mi tan ti reli relo.

Métié du repasseusse,

Mi tan ti reli relo.
Et laquelle prendrez-vous?

Ma tan ti reli relo.

Et laquelle prendrez- vous?

Ma tan ti reli relo.

Nous prendrons $\mathrm{M}^{\text {lle }} \mathrm{X}$,

Ma tan ti reli relo.

Nous prendrons $\mathbf{M}^{\text {lle }} \mathbf{X}$,

Ma tan ti reli relo.

Et quel métier lui donnerez-vous?

Mi tan ti reli relo.

Et quel métier lui donnerez-vous?

Mi tan ti reli relo.

Le métier de repasseuse.

Mi tan ti reli relo.

Le métier de repasseusse,

Mi tan ti reli relo.

$\mathrm{Nu}$ een wiegeliedje, waarin piti natuurlijk kleintje of lieveling betekent. Ook hier geen verschil in de geslachten: ta manman, ta papa.

1

Fè dodo piti ta manman!

Fè dodo piti ta papa!

Si ou paté domi,

Gros chat la qui dan boi la

Ka vini mangé ou.

\section{2}

Fè dodo piti ta manman!

Fè dodo piti ta papa!

Manman ou pa la,

Papa ou pati;

Si ou palé domi,

Gros chat la qui dan bois la

Ka vini mangé ou.
LE SOMMEIL DE L'ENFANT.

Fais dodo chéri de maman!

Fais dodo chéri de papa!

Si tu ne veux pas dormir,

Le gros chat qui est dans les bois

Viendra te manger.

Fais dodo chéri de maman!

Fais dodo chéri de papa!

Ta mère n'est pas là,

Ton père est parti;

Si tu ne veux pas dormir,

Le gros chat qui est dans les bois

Viendra te manger.

En thans een lied met een dramatische inslag. Si betekent suis.

YON PITI MENDIANTE.

1

Cé yon piti mendiante
UNE PETITE MENDIANTE.

C'est une pétite mendiante 
Qui ca mandé ou en moceau pain; Bail à la pòve innocente, Bail, bail car y tini faim.

\section{2}

$\mathrm{Pa}$ rejété priè a moin; Coeur ou kail dirou pouqui;

Moin ni siz an et moin pa ni

Moin faim: ayé pitié du moin. manm
moin.

\section{3}

Hiè cété fète a vilage;

A moin pesonne pa songé;

Chacun té ca joué a sou féyage;

Hélas! et moin pa mangé.

\section{4}

Padonné moin si moin ka mandé, Moin ca mandé rien que du pain, Du, pain, moin pa si gourmande; A! pa grondé moin, moin ni faim.

\section{5}

$\mathrm{Pa}$ couè moin ka ignoré Dan le monde foc ou soufè, Mai moin si piti encô, A pa léssé moin môr.

\section{6}

Bail a la pôvre pitite,

Et pour ou y kail prié.

Y ni faim; bail, bail vite;

Bail, yon note kail renne ou li.
Qui vous demande un morceau de [pain;

Donnez à la pauvre innocente, Donnez, donnez car elle a faim.

Ne rejetez pas ma prière;

Votre coeur vous dira pourquoi; J'ai six ans, je n'ai plus de mère, J'ai faim: ayez pitié de moi.
Hier, c'était fête au village;

A moi personne n'a songé;

Chacum jouait sous le feuillage; Hélas! et je n'ai pas mangé.

Pardonnez-moi si je demande, Je ne demande que du pain, Du pain, je ne suis pas gour[mande

Ah! ne me grondez pas, j'ai faim.

Ne croyez pas que j'ignore Que dans ce monde il faut souf[frir,

Mais je suis si petite encore,

$\mathrm{Ah}$ ! ne me laissez pas mourir.

Donnez à la pauvre petite, Et pour vous elle priera.

Elle a faim; donnez, donnez vite; Donnez, un autre vous le rendra.

Niet minder dramatisch is het volgende kinderlied, waarbij tevens enkele kindernamen en spijzen de revue passeren.

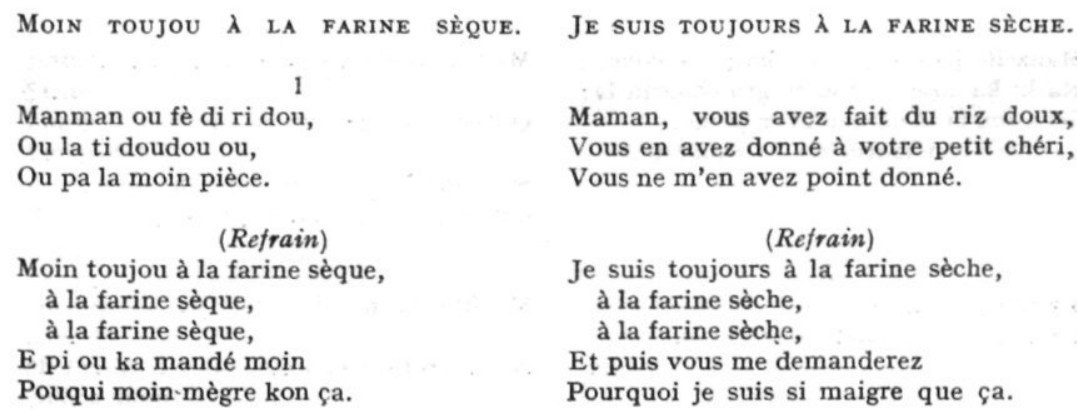

\section{(Refrain)}

Moin toujou à la farine sèque, à la farine sèque,$$
\text { à la farine sèque, }
$$$$
\text { E pi ou ka mandé moin }
$$$$
\text { Pouqui moin-mègre kon ça. }
$$

Maman, vous avez fait du riz doux, Vous en avez donné à votre petit chéri, Vous ne m'en avez point donné.

$$
\text { (Refrain) }
$$

Je suis toujours à la farine sèche,

à la farine sèche,

à la farine sèche,

Et puis vous me demanderez

Pourquoi je suis si maigre que ça. 
Manman ou fè chocola, Ou la ti Colas ou,

Ou pa la moin pièce.

\section{3}

Manman ou fè ti siro, Ou la ti Roro ou,

Ou pa la moin pièce.

\section{4}

Manman ou bouè coco, Ou la ti Cocotte ou,

Ou pa la moin pièce.

5

Manman ou mangé pataté,

Ou la ti doudou ou,

Ou pa la moin pièce.

Manman ou fè du té,

Ou la ti chéri ou,

Ou pa la moin pièce.

$$
7
$$

Manman ou bouè café, Ou la ti Felo ou,

Ou pa la moin pièce.

8

Manman ou mangé zigname,

Ou la ti Cocotte ou,

Ou pa la moin pièce.

9

Manman ou mangé décè,

Ou la ti chéri ou,

Ou pa la moin pièce.

Tot hetzelfde genre behoort:

Manzelle Ka pléré.

Manzelle ka ou ti ni ou ka pléré conça? Ra ki ka cosé ou tou ce gro chagrin la? Sel a sou la tè on peu ni en peine, Car dépi bon matin ou ka sangloté.

Ma fille pa mandé moin ça ca désolé moin, $\mathrm{Pa}$ chêché a connète secré ki en coeu a [moin,
Maman, vous avez fait du chocolat, Vous en avez donné à votre petit Colas, Vous ne m'en avez point donné.

Maman, vous avez fait du petit sirop, Vous en avez donné à votre petit Roro, Vous ne m'en avez point donné.

Maman, vous avez bu du coco, Vous en avez donné à votre petite Vous ne m'en avez point donné. [fillette,

Maman, vous avez mangé des pommes de [terre, Vous en avez donné à votre petit chéri, Vous ne m'en avez point donné.

Maman, vous avez fait du thé,

Vous en avez donné à votre petit chéri, Vous ne m'en avez point donné.

Maman, vous avez bu du café,

Vous en avez donné à votre petit Felo, Vous ne m'en avez point donné.

Maman, vous avez mangé des yams, Vous en avez donné à votre petite Vous ne m'en avez point donné. [fillette,

Maman, vous avez mangé du dessert, Vous en avez donné à votre petit chéri, Vous ne m'en avez point donné.

\section{Mademoiselle pleurera.}

Mademoiselle qu'avez-vous pour pleurer [ainsi? Qu'est ce qui cause tout ce grand [chagrin?

Seul sur la terre je suis en peine,

Car depuis ce matin je sanglote.

Ma fille ne me demande pas ce qui me [désole,

Ne cherche pas à connaître le sécret de [mon coeur, 
La douleur la plu triste vini tracassé [moin.

Hélas! moin déléssé, yo abandoné moin.

$$
3
$$

Yon fleu pa ka jamin resté san yon pa[pillon,

Yon ciel pa ka jamin resté san yon [zétoile,

Yon mer pa ka jamin resté san yon flo. Hélas! moin déléssé, yo abandoné moin.

Ma fille faut pa ou palé con ou ka palé ya, Réfléchi en momen dumoin, moin kaille [sourir ou.

Hélas, moin déléssé, troi jou moin [déléssé.

Moin déléssé passe ça moin inmin la pati.

Hélas! chagrin la ca gagné manzelle, Y ka pléré, pléré san jamin consolé.

Yon jou cé tou bonnement dominus

L'abbé minné li en gran cimitiè la.

$$
\text { [vobiscum, }
$$

$$
6
$$

Hélas, chè! a mon tou moin trapé en [douleu;

Moin chanté chanson a ou té ka pleuré la?

Moin déposé yon rose assou tombo a li;

Moin tanne li ka di: yo abandoné moin!
La douleur la plus triste vient me tra[casser. Hélas! je suis délaissée, on m’a aban[donnée.

Une fleur n'est restée jamais sans un [papillon, Un ciel n'est resté jamais sans une [étoile, Une mer n'est restée jamais sans une [fleuve.

Hélas! je suis délaissée, on m'a aban[donnée.

Ma fille ne faut pas parler comme vous [parlez là, Réfléchiez au moins un moment, je vais [sourir avec vous.
Hélas, je suis délaissée, trois jours je suis [délaissée. Je suis délaissée parce'que celui qui [j'aimais est parti.

Hélas le chagrin a gagné mademoiselle, Elle pleure, elle pleure sans être jamais

[consolée.

Un jour c'est tout à fait dominus [vobiscum,

L'abbé l'emmène dans la grande cime[tière.

Hélas chérie! en mon tour j'ai eu une [douleur;

J'ai chanté votre chanson: qui pleure là ? Je dépose une rose sur son tombeau; Je l'ai entendu dire: on m'a abondonnée!

Er is geen Fransman, of hij heeft zijn lied aan vlag en vaderland, en in die liefde doet de Creool niet voor hem onder met zijn liefde $d u$ patri (de la patrie).

ESCE OU CONNÈTE LÉ TROI COULEे.

\section{1}

Esce ou connète lé troi coulè,

Lé troi coulé de France;

Ça ki ca fè révé lé coeu

En gloire et espérence;

Blé céleste, coulè du jou,

Rouge de san, coulè d'amour,

Blan franchize et vayiance.

$$
2
$$

Jusca la mòr y ka défanne,

\section{LES CONNAIS-TU LES TROIS COULEURS.}

Les connais-tu les trois couleurs, Les trois couleurs de France; Celles qui font rêver les coeurs De gloire et d'espérance;

Bleu céleste, couleur du jour,

Rouge de sang, couleur d'amour, Blanc, franchise et vaillance.

Jusqu'à la mort on les défend, 
Yon sublime folie, Et can yo ka vini trionfé Tou pré étofe chéri la, Toute zieu plein d'larme, Car drapo la ka gadé en pli li L'âme du patri la.
Une sublime folie,

Et quand on revient triomphant Tout près sa loque chérie,

Tous les yeux sont de larmes remplis, Car le drapeau garde en ses plis L'âme de la patrie.

Een der bekendste volksliedjes is Adieu madras, het afscheid van het meisje aan een matroos. De foula is de mooie halsdoek, die evenals de madras, de hoofddoek, en de gouden sierraden tot de Guadeloupse dracht behoren. Colier chou is de halsketting met grote kralen. - Het sou marin, in het vierde couplet, kan ik niet thuis brengen. Het is omgezet in un marin, maar dit zal wel niet goed zijn.

\section{Adieu madras.}

\section{1}

Adieu foula, adieu madras, Adieu grinne dou, adieu colié

Doudou à moin li ka pati. [chou;

Hélas, hélas cé pou toujou!

$$
2
$$

Moin za mandé le Procureu Pou léssé doudou épi moin; Le Procureu fai moin pou réponse: Hélas! hélas y déja tro tar!

\section{3}

Hélas, bon Dieu, la moin la fôce Pou voè doudou moin pati;

Hélas, bon Dieu, la moin courage Pou voè doudou pati tout à l'hêu.

$$
4
$$

Hélas bon Dieu, si moin té sou [marin,

Pou moin té condui doudou moin; Hélas, bon Dieu, si moin té sou [marin,

Pou moin té allé épi doudou moin.

$$
5
$$

Moin ka maudi capitaine la, Ki vini épi batau à li;

\section{AdieU madras.}

Adieu foulard, adieu madras,

Adieu graines d'or, adieu colier

Mon cheri, il s'en va. [chou;

Hélas, hélas c'est pour tou[jours!

J'ai demandé le Procureur Pour laisser mon chéri avec moi; Le Procureur me fit comme [réponse:

Hélas; hélas il est déjà trop tard!

Hélas, bon Dieu, donne moi la [force

Pour voir partir mon chéri; Hélas! bon Dieu, donnez moi le [courage

Pour voir partir tout à l'heure [mon chéri.

Hélas, bon Dieu, si j'étais un [marin,

Pour que je conduisse mon chéri; Hélas, bon Dieu, si j'étais un [marin,

Pour que j'aille avec mon chéri.

Je maudis le capitaine Qui vient avec son bâteau; 
Y pren doudou miné y allé. Hélas, hélas cé pou toujou!

Moin ka maudi bâtiment là

$\mathrm{Ki}$ vini pren doudou moin;

Moin ka maudi bâtiment là

Ki minné doudou moin allé!

PAPA MOIN BA MOI YON MARI.

Papa moin la moin yon mari, mon

[Dieu,

Qui nomme qui piti nomme chè!

Papa moin la moin yon mari, mon

Qui nomme qui li piti!

$$
2
$$

[Dieu,

Moin pren lampe la pou chaché li, [mon Dieu,

Qui nomme qui piti nomme chè!

Moin pren lampe la pou chaché li,

Qui nomme qui li piti! mon Dieu,

$$
3
$$

Du feu pri dan paillasse à li, mon [Dieu,

Qui nomme qui piti nomme chè!

Du feu pri dan paillasse à li, mon

Qui nomme qui li piti!

[Dieu,

\section{4}

Chatte la pren li pou on souri, [mon Dieu,

Qui nomme qui piti nomme chè!

Chatte la pren li pou on souri, mon

Qui nomme qui li piti!

[Dieu,

$$
5
$$

Chatte là, chatte là cé mari à moin, [mon Dieu,

Qui nomme qui piti nomme chè!

Chatte là, chatte là cé mari à moin,

Qui nomme qui li piti!
Il prend mon chéri et l'emmène. Hélas, hélas c'est pour toujours!

Je maudis le bâteau

Qui est venu prendre mon chéri;

Je maudis le bâteau

Qui emmène mon chéri aller!

MON PÈRE M'A DONNÉ UN MARI.

Mon père m'a donné un mari, mon

[Dieu,

Quel homme, quel petit homme

[chéri!

Mon père m'a donné un mari, mon

Quel homme qu'il est petit!

[Dieu,

Je pris la lampe pour le chercher,

Quel homme, quel petit homme

[chéri!

Je pris la lampe pour le chercher, Quel homme qu'il est petit!

Le feu a pris dans sa paillasse, [mon Dieu,

Quel homme, quel petit homme [chéri!

Le feu a pris dans sa paillasse,

Quel homme qu'il est petit!

Le chat l'a pris pour une souris, [mon Dieu,

Quel homme, quel petit homme [chéri!

Le chat l'a pris pour une souris,

Quel homme qu'il est petit!

Au chat, au chat c'est mon mari, [mon Dieu,

Quel homme, quel petit homme [chéri!

Au chat, au chat c'est mon mari, Quel homme qu'il est petit! 
Het is natuurlijk inconsequent on in het vierde couplet van het volgende vers fait te schrijven, waar op andere plaatsen fè gespeld wordt; maar de Creolen zijn nu eenmaal erg willekeurig in hun schrijfwijze. Zott, in de refreinen, betekent eigenlijk vous autres.

SURAH.

Moin pren Surah dan sein à manman li; Moin élivé Surah épi zoeufs et madère; A dix huit ans, yo pren li dan lanmain [moin; Cé la moin di bon Dieu: touté fini pour [moin.

\section{(Refrain)}

Nỏn, non! non non mèdame!

Zott pé ké pren Surah;

Pace quan moin té décidé, Zott po cô té paré.

Surah à moin té ni yon abitude; Tou lé six heû du soir, plani dan bra à [moin; Cé la moin di bon Dieu, voyé Surah la [moin.

$$
3
$$

Surah à moin cé yon bel ti gaçon;

Tou li abillé du noè, y ca samme yon

Surah à moin cé yon bel ti gaçon, papillon;

Tou li abillé du blan, y ca samme un [bébé rose.

4

Moin za passé dan toute lé pharmacies, Et pi moin za mandé sous l'onguent [d'amou;

Les pharmaciens fait moin pou la [réponse:

Cé seul'ment Surah à moin qui pé con[solé moin.

\section{(Dernier refrait)}

Moin vé pas encò

Moin save trouvé en jeune gaçon

Qui peu console moin.
Surah.

J'ai pris Surah du sein de sa mère; J'ai élevé Surah avec des oeufs et du [madère;

A dix-huit on me l'a pris des mains; C'est alors que j'ai dit: Mon Dieu, tout [est fini pour moi.

\section{(Refrain)}

Non, non! non non mesdames

Vous ne prendrez pas Surah

Parce que quand je me suis décidée, Vous n'étiez pas encore(préparées) prêtes.

Mon Surah avait une habitude;

Tous les soirs à six heures, (plani) dans [mes bras);

Six heures a sonné, je ne le vois pas [venir;

C'est alors que je dit: Mon Dieu, envoyez [moi mon Surah.

Mon Surah c'est un joli petit garçon; Habillé tout en noir, il ressemble un [papillon;

Mon Surah c'est un joli petit garçon, Habillé tout en blanc, il ressemble un [bébé rose.

J'ai passé dans toutes les pharmacies, Et puis j'ai demandé de l'onguent [d'amour;

Les pharmaciens m'ont fait (donner) [pour réponse: C'est seulement mon Surah qui peut me [consoler.

\section{(Dernier refrain)}

Prenez-le, prenez-le pour vous (autres); Je n'ai pas encore vu

Je peux trouver un jeune garçon

Qui peut me consoler.

In het eerstvolgende couplet staat marabout waarschijnlijk wel in de betekenis van een lelijk en kreupel mens. Chandelle is hier kaars. Grasse is mollig. 


\section{Choucoune.}

Choucoune cé yon maraboute;

Zieu li cléré quon chandel;

Li pa gro femme mé li grassette.

Femme con ça plè moin tou nête

Nou rété cosé lontan. (bis)

\section{(Refrain)}

Ti zozio dan bois qui ta pé couté Quan moin songé ça (bis)

Moin gagné la pinne,

Car dépi jou la (bis)

Dé pié moin dan chinne.

$$
2
$$

Allé la caille manman li

Yon gran moune qui bien onête Sito li vouè moin li di:

A ta la plè moin tou nête.

Nou bouè chocola au noî. (bis)

Meuble prê, bel cabanne bato;

Table ronde, chèse ronde dodine;

Nape, serviette, rido mousline;

Dra brodé et bel tapi.

Quinze jou seilmen té rété. (bis)

4

Yon ti blan vini rivé,

Monte ci côté bo chivé;

Malè moin qui fi la côse,

Li trouvé Choucoune jol

Et Choucoune immé li.

\section{O Casino faIt MOIN PLÉsi.}

1

Dépi moin contré ou,

Dépi ou ranne moin fou,

Dépi cé ou tou seul

Moin ka trouvé ki bel.

\section{(Refrain)}

Min cé o casino ou ka fai moin

[plési

Min bigine là ka cho aucite ou

[pati.
Сhoucoune.

Choucoune c'est un marabout;

Ses yeux brillent comme une chandelle;

Elle n'est pas une grosse femme, mais

$$
\text { [elle grasse. }
$$

Une telle femme me plait tout à fait

Nous sommes restés en causant long-

[temps.

\section{(Refrain)}

Les oiseaux dans les bois qui peuvent m'écouter

Quand j'ai pensé à ça (bis)

J'ai eu de la peine,

Car depuis ce jour là (bis)

Mes deux pieds sont dans une chaine.

J'ai été à la maison de sa mère,

Une grande personne, qui est bien [honnête;

Aussitôt qu'elle m'a vu elle a dit:

A celle là me plaît tout à fait.

Nous avons bu du chocolat au noir. (bis)

Meubles prêts, une jolie cabans de [bâteau;

Une table ronde, une chaise ronde [pour se dodiner;

Une nappe, serviette, des rideaux de [mousseline;

Des draps brodés et un joli tapis.

Quinze jours seulement j'y suis resté. (bis)

Un petit blanc arrive,

Monte de ce côté beaux cheveux;

Mon malheur voilà la cause,

Il a trouvé Choucoune jolie

Et Choucoune l'aime.

\section{LE CASINO ME FAIT PLAISIR.}

Depuis que je vous ai rencontrée, Depuis que vous m'avez rendu fou, Depuis que c'est vous toute seule Que je trouve si belle (jolie).

\section{(Refrain)}

Mais c'est au casino (= badhotel) [que vous me faites plaisir Mais la biguine (= dans) chauffé [aussitôt que vous étiez partie. 
$\mathrm{Pa}$ ti ni pa yon femme qui ka densé comme vous,

Min ça ki belle moune cé ou.

$$
2
$$

Soleil en moin cé ou!

Toute moune jalou nou.

Min ça moin ka di ou,

$\mathrm{Pa}$ léssé moune palé mal di ou.

$$
3
$$

Min qui soi robe encò

Evè foula o ven,

Qui soi colié dò

Qui soi robe di chamme blan.
Il n'y a pas une femme qui danse [comme vous, Mais c'est vous qui êtes une belle [personne.

Vous êtes mon soleil!

Tout le monde est jaloux de nous. Mais ce que je vous dis,

$\mathrm{Ne}$ laissez personne parler mal de

[vous.

Mais que se soit une robe encore Enfin jette la au vent,

Que se soit un collier d'or

Que se soit une robe de chambre

[blanche.

In het lied van de Congolees komen wij nog een andere vorm van un tegen, namelijk yonne. - Tafia is rhum, de volksdrank.

\section{Chant D'Un congo.}

$\mathrm{Y}$ ti ni troi zomme

Qui assou na tabe,

Y ti ni dé ki ti ni den,

Ka mangé pain à pi fômage;

Yonne qui pa ti ni den,

Ka boué divin à pi tafia.

Chanté bien Naxiome,

Chanté bien.

Cé pa moin qui ti fai

La chanson, cé ou minne

$\mathrm{Ki}$ ti fai la chanson ou.

$$
3
$$

Cé pa moin qui ti fai

La chanson,

Cé linton qui fai la chanson.
Il ya trois hommes

Qui sont à table,

Il y en a deux qui ont des dents,

Ils mangent du pain et puis du [fromage;

Il y en a un qui n'a pas de dents, Il boit du vin et puis du tafia.

Chantez bien Naxiome,

Chantez bien.

Ce n'est pas moi qui a fait

La chanson, c'est vous même

Qui a fait votre chanson.

Ce n'est pas moi qui a fait

La chanson,

C'est le temps qui fait la chanson.

Het volgende liedje is een plaatselijk spotliedje; nu weer vergeten, maar gezongen in de tijd van het huwelijk van Lélia en Heribert, dat al direct een mislukking was, aangezien de bruid het huis in de huwelijksnacht ontvluchtte. Vandaar natuurlijk de volksuitleg, dat Lélia, geprest door haar moeder, Heribert puur en alleen om zijn geld trouwde. C'est affaire à Lélia; dat moet Lélia maar weten! 


\section{Maman Lélia.}

1

Maman Lélia mayé Lélia

C'est pou trapé bel maison là.

En arrivant dans maison li

Bonhêu Lélia fini pou li.

\section{(Refrain)}

Zaffai' cor Lélia,

Zaffai' cor à maman Lélia,

Zaffai' cor Lélia,

Zaffai' cor à maman Lélia.

2

Maman Lélia mayé Lélia

Cé pour trapé bijouterie là

Missié Héribert té ni caché.

Hélas, moin di ou toute moune

[sauvé.

3

Premier qui sauvé de case là

C'est maman, mamzelle Lélia.

En arrivant dans maison là

Mamzelle Lélia sauté funêtre là.
Maman Lélia.

Maman Lélia a marié Lélia

C'est pour avoir la jolie maison. En arrivant dans sa maison Le bonheur de Lélia était fini pour [elle.

C'est affaire à Lélia,

C'est affaire à maman Lélia,

C'est affaire à Lélia,

C'est affaire à maman Lélia.

Maman Lélia a marié Lélia

C'est pour obtenir (attraper) les [bijoux

Que Monsieur Héribert avait de [cachés.

Hélas, je vous dis tout le monde

[s'est sauvé.

La première qui s'est sauvée de la rmaison

C'est maman, mademoiselle lélia.

En arrivant dans la maison

Mademoiselle Lélia a sauté par la

[fenêtre

\section{KINDERSPELEN.}

De meeste van deze liedjes worden gezongen, waarbij de kinderen in een kring aftellen.

Si lapin vini ba li bouè,

ba li mangé pa ba li la

clé bufé la, passe si yon

ti bète ki tro malin, lin lin, colin la plu bel la min dého.

Mi yonne, mi dé,

mi dlo chale babi,

babi cano mété

dlo dan la chapel.
Si le lapin vient, donne lui à boire, donne lui à manger, ne lui donne [pas la

clef du buffet, parce' que c'est une petite bête, qui est trop maligne, colline la plus belle la main [(l'emmène?) dehors.

Voici un, voici deux,

voici l'eau chaud,

.... cano mettre

de l'eau dans la chapelle.

Is chale hierbij een verbastering van chaud? Of moet het Charles betekenen, waarbij Charles Babi een kindernaam zou zijn. En dan 
cano mété. Is dat een herinnering aan mettre à la voile, onder zeil gaan, te meer daar het hele geval nogal waterachtig is ?

Pou lavé lé ban de Dieu,

Pour laver le ban de Dieu, pin, pin karabel, la plu pin, pin karabel, la plus bel la min dého. belle la main (l'emmène?) dehors.

Wat mag le ban de Dieu hier wel betekenen?

Pimpomme, balancé, coq chanté, palillon volé.

Ou pa conète za fêta la ou pa save sa ki la, allé chèchè ti brin dlo la main, pou moin lavé pié moin.
Pimpomme (?) balance, le coq chante, le papillon vole.

Vous ne connaissez pas cette [affaire là,

vous ne savez pas ce qu'il y a, va chercher un peu d'eau pour moi, pour me laver les pieds.

RAADSELS

Niet alleen liedjes, maar ook raadsels nemen een belangrijke plaats in in het leven van de Creolen.

Dlo douboute.

De l'eau debout.

\section{Canne d sucre.}

Dlo descen ne mône.

L'eau qui descend un morne.

Un coco.

(Kokosnoten die door ratten zijn aangevreten gaan dikwijls lekken, waarbij het water op de grond druipt).

Moin ici, moin en France.

$$
\text { Une lettre. }
$$

Je suis ici, je suis en France.

Tablier madanme dêyè do i. Le tablier de madame est derrière Un ongle.

[son dos.

Yon case a sou yon sel poté. Une maison sur un seul poteau. Un parapluie

Madanme en lécuri la ké i dérô. Madame à l'écurie sa queue est Cheval. 
Yon ti bononme ka plein en case. Un bonhomme qui remplit une

Une lampe.

[maison.

Moin pa ni roi, ni reine,

Je ne suis ni roi ni reine,

moin ka pôté la couronne. je porte la couronne.

Grenade (granaatappel).

Moin ka tanne vié femme ka J'entends quelques vieilles fem[babiyé, mes se disputer. moin ka gadé, moin pa ka vouè a je regarde et je ne vois rien.

[yen.

Haricots secs.

(Als de snijbonen droog zijn hoor je de boontjes in de basten rammelen).

Madanme voyé servante en con- Madame envoie sa servante faire mission, conmission rivé avant ser- des commissions et les commisvante. sions arrivent avant elle.

Monteur de cocos.

(Iemand die cocosnoten plukt, en dus de palm beklimt, gooit de noten naar beneden vóórdat hijzelf weer omlaag komt).

Moin ka maché, moin ka vouê yon Je marche et je vois un bâton ti baton verni a tèla moin passa verni à terre et ne peux le rapren li. masser.

Un serpent.

Moin ni yon bitin a case moin, J'ai chez moi une chose, toutan moin pa kinbé i pa cou y pa ka sèvi moin. Potiche. qui me sert que lorsque je la prends par le cou.

(Bitin betekent chose; sèvi is eigenlijk servir).

Als een soort s preekw oorde n kunnen gelden:

Rata passa cassa di vers et si ta. Le rat passe et casse dix vers et six tasses.

Si cé paté vini vinette dormi, Si le chien n'était pas arrivé, dormette paté kail mangé tombi le cochon n'aurait pas mangé le
[tombette. corosol.

De laatste uitdrukking is niet zo gemakkelijk thuis te brengen; zij wordt gedragen op een soort van binnenrijm: vini vinette en tombi tombette. Een corosol is een vrucht welke op Curaçao schubappel heet, de sweetapple van de Engelse eilanden. 
En tot slot dat wat men op Guadeloupe zingt om met regen mooi weer te krijgen:

Ange Gabriel pren la pluie, voyé soleil.
Ange Gabriel prend la pluie, envoyez le soleil.

Wordt op Martinique gezongen als:

Missié Coco pren la pluie, voyé soleil.

Monsieur Coco prend la pluie, envoyez le soleil.

\section{R É S U M É}

\section{Chansons Français-Créoles de la Guadeloupe.}

Voici une petite collection de chansons créoles folkloriques, qui sont chantées en Guadeloupe.

Quelques unes de ces chansons sont des jeux enfantins et cette collection est augmentée de quelques énigmes et expressions.

Les Créoles possèdent quelques mots d'eux-mêmes (case-maison, bail ou ba-donner), mais généralement ils usent les mots courants mais ils ont le penchant de simplifier la prononciation et aussil'orthographie; comme: couè-croire, cò-corps, pède-perdre.

A cause de la même nonchalance et par ignorance ils ne distinguent guère les genres des mots et le singulier et le pluriel (en jeune fiyète, du patrie, $d u$ go ans).

Le verbe être chez eux est: $t i, t e, t i n i$; une forme qu'ils usent sans conjugaison (je suis, il est, nous sommes, il était).

Pour indiquer le futur ils usent: ca ou kaille (ca mor-elle mourra).

Pour le pronom personnel il et elle ils emploient $y$.

Le pronom possessif est moin pour mon, ma ou mes et $l i$ pour son, sa et ses. Il est placé après le substantif (tête li-sa tête; jeune an moin- mes jeunes ans). Mais moin peut être aussi le pronom personnel: moin-je.

L'article indéfini un et une est an, on, en, yon, yonne.

Les chansons parlent pour elles-mêmes, seulement „Maman Lélia” demande une petite explication. C'est une chanson locale exprimant une certaine raillerie. Lélia obligée par sa mère se marie à contre coeur avec Monsieur Héribert, mais le soir même du mariage elle s'enfuie. C'est pourquoi l'explication des gens: Lélia a epousé Héribert à cause de son argent.

De cette façon, on voit que les Créoles expriment, tout en chantant, leurs sentiments de tristesse et de joie, mais la plupart de ces chansons ont un caractère mélancolique. 